\title{
Molecular characterization of group A rotavirus before and after the introduction of vaccines in Brazil
}

\section{Tâmera Nunes Vieira Almeida ${ }^{[1]}$, Fabíola Souza Fiaccadori ${ }^{[1]}$, Menira Souza ${ }^{[1]}$, Ana Maria Tavares Borges ${ }^{[1]}$ and Divina das Dôres de Paula Cardoso ${ }^{[1]}$}

[1]. Departamento de Microbiologia, Imunologia, Parasitologia e Patologia Geral, Instituto de Patologia Tropical e Saúde Pública, Universidade Federal de Goiás, Goiânia, Goiás, Brazil.

\begin{abstract}
Introduction: In this study, the molecular characteristics of group A rotavirus (RVA) were compared in samples obtained before and after RVA vaccine-introduction in Brazil. Methods: Eighty samples were screened for the presence of RVA. Positive samples were molecularly analyzed. Results: RVA positivity was $16.9 \%$, with a predominance of G2P[4]. Periods: pre-vaccination: predominance of IId (G1), IId (G2) lineages, and I1 and E1 genotypes; post-vaccination: predominance of Ib (G1), IIa, and IIc (G2) lineages and I2 and E2 genotypes. Conclusions: Although changes in RVA-circulation pattern were observed in the post-vaccination period, it could not be attributed to vaccination process.
\end{abstract}

Keywords: Rotavirus A. Gastroenteritis. Vaccine.

Group A rotaviruses (RVAs) are among the most important agents of acute gastroenteritis in humans and are responsible for significant morbidity worldwide, with high mortality rates being reported in developing countries. RVAs are non-enveloped, triple-layered particles that enclose 11 genomic segments of double-stranded ribonucleic acid (RNA). The viral genome encodes 12 proteins, including 6 structural (VP1-VP4, VP6 and VP7) and 6 non-structural (NSP1-NSP6) proteins. The outer capsid layer is formed by the VP7 and VP4 proteins, which elicit neutralizing antibodies. The middle layer is composed of VP6, which also has antigenic and immunogenic properties. The inner layer is constituted of VP2 that, together with VP1-VP3, surrounds the genome. The non-structural proteins are involved in viral morphogenesis, particularly NSP4, which potentially has enterotoxigenic properties ${ }^{(1)}$.

Considering the burden of infections caused by RVA, 2 vaccines, Rotarix ${ }^{\circledR}$ (GlaxoSmithKline) and RotaTeq ${ }^{\circledR}$ (Merck Sharp \& Dohme), were licensed and have been available for use since 2006. Rotarix ${ }^{\circledR}$ is a monovalent human rotavirus vaccine that was introduced through the National Immunization Program (NIP) of Brazil in March 2006 ${ }^{(2)}$. RotaTeq ${ }^{\circledR}$ is a pentavalent bovine-human reassortant rotavirus vaccine that is also available in private immunization clinics in the country.

Corresponding author: Dra. Divina das Dôres de Paula Cardoso. Rua $235 \mathrm{~s} / \mathrm{n}$, esquina com 1a. Avenida, Setor Universitário, 74605-050 Goiânia, Goiás, Brasil. Phone: 5562 3209-6244; Fax: 5562 3209-6363

e-mail: dcardoso@ufg.br

Received 23 January 2015

Accepted 14 May 2015
In this study, we investigated RVA positivity in children presenting with gastroenteritis during 2008-2009 and conducted comparative molecular analysis with positive samples from pre- and post-vaccination periods, considering the VP7, VP6, and NSP4 genes.

The study material consisted of 80 stool samples from children who attended either of two hospitals (Materno Infantil Hospital and Pronto Socorro Infantil) in Goiânia, Goiás, Brazil. The samples were all from children less than 5 years old with symptoms of acute gastroenteritis, with or without vomiting, fever, abdominal cramps, or nausea for at least 24 hours. This study cohort included patients that were or were not vaccinated against RVA, and request hospitalization.

Of the 80 samples studied, 65 were collected during July 2008-December 2009, and 15 were archived samples from the Laboratory of Human Virology of Institute of Tropical Pathology and Public Health/Federal University of Goiás. The archived samples were obtained during 1992, 2005, and 2006 and were previously genotyped for the presence of the RVA VP7 and VP4 genes $^{(3)(4)}$. The fecal samples obtained during 20082009 were stored at $-20^{\circ} \mathrm{C}$ until fecal suspensions were prepared in phosphate buffered saline $(20 \%, \mathrm{w} / \mathrm{v})$. The archived samples were stored at $-80^{\circ} \mathrm{C}$ until use. This study was approved by the Ethics in Research Committee at the Materno Infantil Hospital (Protocol 006/08), and in accordance with the ethical standards of the national committee involving human [Comissão Nacional de Ética em Pesquisa (CONEP)], Resolution 466/12.

Group A rotavirus was detected in stool samples using a commercial enzyme immunoassay (EIA) kit (the RIDASCREEN ${ }^{\circledR}$ Rotavirus Kit; R-Biopharm AG, 
Darmstadt, Germany) and by polyacrylamide gel electrophoresis (PAGE). Group A rotavirus-positive samples were genotyped for VP7 and VP4 by the polymerase chain reaction (PCR), using a published primer set and protocol ${ }^{(5)(6)}$. VP6 and NSP4 gene amplification was performed as described previously ${ }^{(7)(8)}$.

For genomic sequencing, PCR products were purified using the QIAquik ${ }^{\circledR}$ PCR Purification Kit (Qiagen, Valencia, $\mathrm{CA}$ ) and sequenced using the PCR primers. All samples were sequenced in an ABI Prism 3130 Genetic Analyzer (Applied Biosystems, Foster City, CA, USA). The VP6 and NSP4 gene sequences with good-quality reads were aligned using Clustal $\mathrm{X}$, and phylogenetic trees were constructed with MEGA6, using the neighbor-joining algorithm and a bootstrap value of 2,000 replicates.

Comparative analysis between the deduced amino acids sequences of the Rotarix ${ }^{\circledR}$ strains [GenBank Accession Numbers: JN849114 (VP7), JX943613 (VP6), and JX943606 (NSP4)] and those in this study was performed using MEGA6 software. The sequences obtained were deposited as GenBank Accession Numbers KJ434169-KJ434173 (VP7), KJ394458KJ394469 (VP6), and KJ206621-KJ206629 (NSP4).

Statistical data analysis was performed with Epi info software, version 3.5.4 (Centers for Disease Control and Prevention, Atlanta, GA), using the chi-squared $\left(\chi^{2}\right)$ test and Fisher's exact test, when appropriate. Statistical significance was assessed at a $\mathrm{p}$ value of $<0.05$.

The positivity rate observed for RVA by EIA and PAGE was $16.9 \%(11 / 65)$ for the samples obtained during 2008-2009. No statistical significances were observed between the positivity rates and gender $(p=0.7418)$, age $(p=0.5895)$, symptoms $(p=0.2416)$, or vaccination status $(p=0.6793)$, although a higher detection rate was detected among unvaccinated children (23.1\%) compared to vaccinated children (15.4\%). Of the 11 positive samples, 4 were characterized as G2P[4], 6 were G2PNT (not genotyped), and 1 was GNTP[9][11].

Table 1 shows that of the 6 archived samples obtained before the introduction of the vaccine in Brazil, 5 were characterized as G1P[8] and 1 was G2PNT. Of the 9 samples collected after the vaccination period, 1 was G2P[8], 1 was G1PNT, and 7 were G2P[4].

Of 26 positive samples subjected to genome sequencing, 16,24 , and 21 had sufficiently high-quality sequences for phylogenetic analysis of the VP7, VP6, and NSP4 genes, respectively. Phylogenetic analysis of the VP4 gene was not possible due to the poor quality of the sequences obtained. $\mathrm{G} 1$ and $\mathrm{G} 2$ samples obtained before introduction of the vaccine belonged to lineage IId. For samples obtained during the postvaccination period, sample $\mathrm{G} 1$ was belonged to lineage $\mathrm{Ib}$, and for the G2 samples, 5 were IIa and 6 were IIc. Considering the VP6 gene, 5 samples from the pre-vaccination period belonged to genotype I1. For the samples obtained during the postvaccination period, 2 were genotype I1 and 17 were genotype I2. For the NSP4 gene, it was observed that 5 samples from the pre-vaccination period belonged to the E1 genotype. For the samples obtained from the post-vaccination period, 2 samples were characterized as E1 genotypes, and 14 were characterized as E2.

Phylogenetic analysis showed that all sequences clustered closely, with an identity ranging from $95.4 \%$ to $100 \%$ (not shown). Comparative amino acid sequence analysis of VP7, VP6, and NSP4 with the corresponding sequences of the vaccine strain (Rotarix ${ }^{\circledR}$ ) showed no significant differences (not shown).

TABLE 1 - Molecular profiles of 26 RVA samples (VP7, VP6, and NSP4 genes).

Genomic sequencing

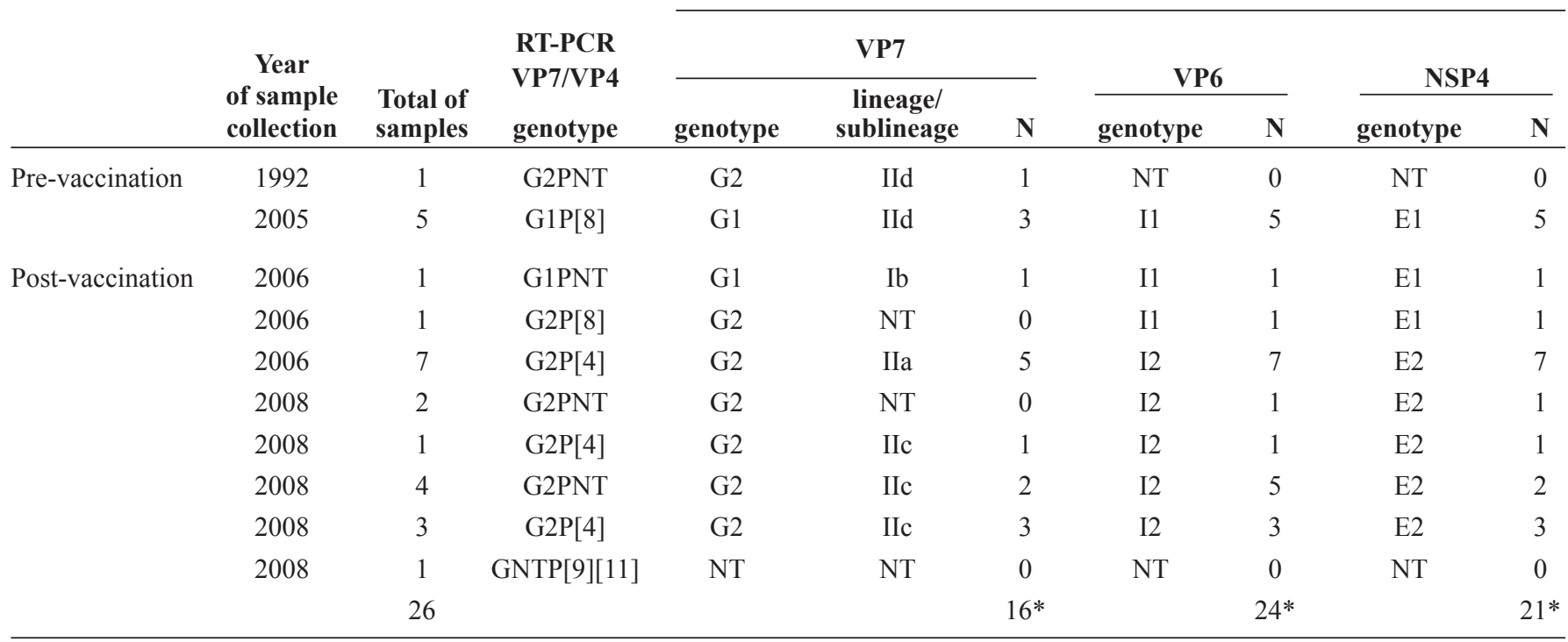

RVA: group A rotavirus; RT-PCR: reverse transcriptase-polymerase chain reaction; N: number of sequences with quality for analysis; NT: not typeable.

*Total number of samples that were sufficient quality for sequence analysis. 
In this study, the positivity rate for RVA was $16.9 \%$, which was lower than previously described ${ }^{(4)}$. Positivity for RVA was observed only in samples collected during 2008, with no positivity observed in samples collected during 2009. This decrease in RVA positivity in the post-vaccination period has been related to the introduction of the RVA vaccines in other studies ${ }^{(2)(9)}$.

The predominance of the G1P[8] genotype was described before the introduction of RVA vaccines in $\mathrm{Brazil}^{(3)}$. In the present study, most RVA samples obtained in the postvaccination period were G2P[4], which is in accordance with a study conducted in Brazil that showed a gradual increase in the detection of G2P[4] samples between 2006 and $2008^{(2)(4)(9)}$.

The predominance of $\mathrm{G} 2$ genotype in this period coincides with the introduction of the RVA vaccine in Brazil. Similar results have been observed in countries from Latin America that implemented RVA vaccines, as well as in countries that have not implemented these vaccines or have a low vaccination coverage ${ }^{(2)}$. Thus, the re-emergence of the $\mathrm{G} 2$ genotype cannot be attributed to vaccine pressure since a cyclic pattern of distinct RVA strains has also been observed by others ${ }^{(2)(3)}$.

Phylogenetic analysis of the VP7 gene showed that the occurrence of intra-genotypic RVA variability with several variants was closely associated with Brazilian samples obtained in both the pre- and post-vaccination periods.

For G1 genotype samples, the occurrence of lineages IId in the pre-vaccination period and $\mathrm{Ib}$ in the post-vaccination period for G1 samples was observed. The circulation of G1 lineages I and II has been previously reported in samples obtained during the pre-vaccination period in Brazil, highlighting the genetic diversity of this genotype ${ }^{(10)}$. However, samples obtained in a recent Brazilian study before and after the beginning of RVA vaccination showed that, although there is great genetic variability in the VP7 gene, such variability has not resulted in diversity in the antigenic sites of this protein ${ }^{(11)}$.

For G2 samples, the occurrence of lineages IId (prevaccination period), as well as IIa and IIc (post-vaccination period) was observed. This pattern was also observed in the northern region of Brazil ${ }^{(12)}$. This alternating pattern may be associated with drifts over time, resulting from natural selection rather than a vaccine-induced, selective-pressure phenomenon.

The VP6 and NSP4 genes were detected in all samples from the pre-vaccination period and were characterized as I1 and E1 genotypes, respectively. In contrast, the samples obtained during the post-vaccination period were predominantly of genotypes I2 and E2. All sequences showed a high identity with Brazilian samples obtained before and after the beginning of RVA vaccination. This pattern of circulation of the VP6 and NSP4 genotypes has been also observed in other studies conducted in Brazil ${ }^{(13)(14)}$.

Analysis considering the VP7, VP4, VP6, and NSP4 genes showed the occurrence of an association G1-P[8]-I1-E1 in the pre-vaccination period with the predominance of G2-P[4]-I2-E2 in the post-vaccination period. These data corroborate previous studies conducted in Brazil before and after the introduction of RVA vaccines ${ }^{(13)(14)}$.
In this study, the occurrence of 1 G2-P[8]-I1-E1 sample was observed, which was collected 3 months after the introduction of the RVA vaccine in Brazil. Sample G2P[8] was also described in a previous study, the authors of which suggested that G2P [8] could be a reassortment between G1P[8] and G2P[4], arising from the co-circulation of these samples ${ }^{(15)}$.

One $\mathrm{P}[9] \mathrm{P}[11]$ sample was detected, which was interesting because both genotypes are usually detected in animals. Furthermore, the occurrence of unusual RVA genotypes have also been reported for studies conducted in Brazil in the post-vaccination period, which appeared to be RVA transmission interspecies ${ }^{(9)}$.

We also conducted a comparative analysis of the sequences of the Rotarix ${ }^{\circledR}$ vaccine genes with our samples obtained from the pre- and post-vaccination periods, and no significant differences were observed in the corresponding amino acid sequences.

A limitation of this study was the small number of fecal samples that were analyzed. Considering the period from 2008 to 2009 , this could be partially attributed to the efficacy of vaccination that resulted in a decreased number of gastroenteritis cases $^{(2)(9)}$. This possibility is reinforced by the fact that sample collection was performed in 2 referral hospitals for pediatric care. The reason for the small number of pre-vaccination samples used in the study is that they were archived samples, and a relatively low quantity of material was available for analysis. Another limitation of this study is related to the phylogenetic analysis of the VP4 gene, which could not be performed due to the quality of the sequences obtained.

In conclusion, we suggest that the data presented in this study reflects change in the RVA circulation patterns in relation to the VP7, VP4, VP6, and NSP4 genes, from samples obtained in the pre and post-vaccination periods. However, these changes could not be directly attributed to RVA vaccination, due to natural fluctuations of these genotypes that occur naturally over time. However, the findings presented here reinforce the importance of continuous monitoring of RVA samples when evaluating the role of vaccination in genotype-pattern changes in RVAs.

\section{CONFLICT OF INTEREST}

The authors declare that there is no conflict of interest.

\section{FINANCIAL SUPPORT}

Conselho Nacional de Desenvolvimento Cientifico e Tecnológico (CNPq) - (Process 484327/2007-10).

\section{REFERENCES}

1. Estes MK, Kapikian AZ. Rotaviruses. In: Knipe DM, Howley PM, editors. Fields Virology. Philadelphia: Wolters Kluwer Health/ Lippincott Williams \& Wilkins; 2013. p. 1347-1401.

2. Linhares AC, Justino MCA. Rotavirus vaccination in Brazil: effectiveness and health impact seven years post-introduction. Expert Rev Vaccines 2014; 13:43-57. 
3. Cardoso das D, Soares CM, Azevedo MS, Leite JP, Munford V, Rácz ML. Serotypes and subgroups of rotavirus isolated from children in central Brazil. J Health Popul Nutr 2000; 18:39-43.

4. Munford V, Gilio AE, de Souza EC, Cardoso DM, Cardoso DD, Borges AM, et al. Rotavirus gastroenteritis in children in 4 regions in Brazil: a hospital-based surveillance study. J Infect Dis 2009; 200 (suppl I):106-113.

5. Gouvea V, Glass RI, Woods P, Taniguchi K, Clark HF, Forrester $\mathrm{B}$, et al. Polymerase chain reaction amplification and typing of rotavirus nucleic acid from stool specimens. J Clin Microbiol 1990; 28:276-282.

6. Gentsch JR, Glass RI, Woods P, Gouvea V, Gorziglia M, Flores J, et al. Identification of group A rotavirus gene 4 types by polymerase chain reaction. J Clin Microbiol 1992; 30:1365-1373.

7. Iturriza-Gómara $\mathrm{M}$, Wong $\mathrm{C}$, Blome $\mathrm{S}$, Desselberger $\mathrm{U}$, Gray J. Molecular characterization of VP6 genes of human rotavirus isolates: correlation of genogroups with subgroups and evidence of independent segregation. J Virol 2002; 76:6596-6601.

8. Lee CN, Wang YL, Kao CL, Zao CL, Lee CY, Chen HN. NSP4 gene analysis of rotaviruses recovered from infected children with and without diarrhea. J Clin Microbiol 2000; 38:4471-4477.

9. Dulgheroff ACB, Figueiredo EF, Moreira LP, Moreira KC, Moura LMS, Gouvêa VS, et al. Distribution of rotavirus genotypes after vaccine introduction in the Triângulo Mineiro region of Brazil: 4-Year follow-up study. J Clin Virol 2012; 55:67-71.
10. Maranhão AG, Vianez-Júnior JL, Benati FJ, Bisch PM, Santos N. Polymorphism of rotavirus genotype G1 in Brazil: in silico analysis of variant strains circulating in Rio de Janeiro from 1996 to 2004. Infect Genet Evol 2012; 12:1397-1404.

11. Silva MFM, Rose TL, Gómez MM, Carvalho-Costa FA, Fialho AM, Assis RMS, et al. G1P[8] species A rotavirus over 27 years - Pre- and post-vaccination eras - in Brazil: Full genomic constellation analysis and no evidence for selection pressure by Rotarix ${ }^{\circledR}$ vaccine. Infect Genet Evol 2015; 30:206-218.

12. Mascarenhas JD, Lima CS, de Oliveira DS, Guerra SF, Maestri RP, Gabbay YB, et al. Identification of two sublineages of genotype G2 rotavirus among diarrheic children in Parauapebas, Southern Pará State, Brazil. J Med Virol 2010; 82:712-719.

13. Gómez MM, de Mendonça MC, Volotão EM, Tort LF, da Silva MF, Cristina J, et al. Rotavirus A genotype P[4]G2: genetic diversity and reassortment events among strains circulating in Brazil between 2005 and 2009. J Med Virol 2011; 83:1093-1106.

14. Tavares TM, Brito WM, Fiaccadori FS, Parente JA, Costa PS, Giugliano LG, et al. Molecular characterization of VP6-encoding gene of group A human rotavirus samples from central west region of Brazil. J Med Virol 2008; 80:2034-2039.

15. Iturriza-Gómara $\mathrm{M}$, Isherwood $\mathrm{B}$, Desselberger $\mathrm{U}$, Gray J. Reassortment in vivo: driving force for diversity of human rotavirus strains isolated in the United Kingdom between 1995 and 1999. J Virol 2001; 75:3696-3705. 